\title{
Preoperative Midregional Pro-Adrenomedullin and High-Sensitivity Troponin T Predict Perioperative Cardiovascular Events in Noncardiac Surgery
}

\author{
Mlađjan Golubovića Radmilo Jankovića, b Dušan Sokolović ${ }^{b}$ \\ Vladan Ćosićc Vera Maravić-Stojkovic ${ }^{d}$ Tomislav Kostićb, e Zoran Perišićb, e \\ Nebojša Lađevićf, $g$ \\ ${ }^{a}$ Center for Anesthesiology and Reanimatology, Clinical Center Nis, Niš, Serbia; ${ }^{b}$ Faculty of Medicine, \\ University of Nis, Niš, Serbia; ${ }^{c}$ Center for Biochemistry, Clinical Center Nis, Niš, Serbia; ${ }^{d}$ Institute for Cardiovascular \\ Diseases, Belgrade, Serbia; ${ }^{\mathrm{e} C l i n i c}$ for Cardiovascular Diseases, Clinical Center Nis, Niš, Serbia; ${ }^{f}$ Center for \\ Anesthesiology and Reanimatology, Clinical Center of Serbia, Belgrade, Serbia; ${ }^{9}$ Faculty of Medicine, University of \\ Belgrade, Belgrade, Serbia
}

\section{Significance of the Study}

- This study evaluated the utility of preoperative midregional pro-adrenomedullin and high-sensitivity troponin $\mathrm{T}$ for improved detection of patients at high risk for perioperative cardiac events and mortality after major noncardiac surgery. Compared to high-sensitivity troponin T, midregional pro-adrenomedullin was more effective as a predictor of perioperative cardiovascular complications.

\section{Keywords}

Midregional pro-adrenomedullin · High-sensitivity troponin P Periooperative complications

\begin{abstract}
Objective: We evaluated the utility of preoperative midregional (MR) pro-adrenomedullin (proADM) and cardiac troponin $\mathrm{T}(\mathrm{TnT})$ for improved detection of patients at high risk for perioperative cardiac events and mortality after major noncardiac surgery. Subjects and Methods: This prospective, single-center, observational study enrolled 79 patients undergoing major noncardiac surgery. After initial clinical assessment (clinical history, physical examination, echocar-
\end{abstract}

diogram, blood tests, and chest X-ray), MR-proADM and high-sensitivity $\mathrm{TnT}$ (hsTnT) were measured within $48 \mathrm{~h}$ prior to surgery by immunoluminometric and electrochemiluminescence immunoassay. Patients were followed by the consulting physician until discharge or up to 14 days in the hospital after surgery. Perioperative cardiac events included myocardial infarction and development or aggravation of congestive heart failure. Data were compared between patients who developed target events and event-free patients. Results: Within 14 days of monitoring, 14 patients (17.72\%) developed target events: 9 (11.39\%) died and 5 (6.33\%) developed cardiovascular events. The average age of the patients was $71.29 \pm 6.62$ years (range: $55-87$ ). Sex, age, and hsTnT did not significantly differ between groups. MR-

\begin{tabular}{ll}
\hline KARGER & $\begin{array}{l}\text { ( ) } 2018 \text { The Author(s) } \\
\text { Published by S. Karger AG, Basel }\end{array}$ \\
E-Mail karger@karger.com & $\begin{array}{l}\text { This is an Open Access article licensed under the Creative Commons } \\
\text { Attribution-NonCommercial-4.0 International License (CC BY-NC) } \\
\text { (http://www.karger.com/Services/OpenAccessLicense), applicable to } \\
\text { the online version of the article only. Usage and distribution for } \\
\text { commercial purposes requires written permission. }\end{array}$
\end{tabular}

Mlađan Golubović

Center for Anesthesiology and Reanimatology

Clinical Center Nis, Grčka 17

RS-18000 Niš (Serbia)

E-Mail mladjangolubovic@gmail.com 
proADM concentration was higher in deceased patients ( $p=$ 0.01 ). The upper quartile of MR-proADM was associated with a fatal outcome $(66.7$ vs. $20.0 \%, p<0.01)$ and with cardiovascular events ( 64.3 vs. $16.9 \%, p<0.01)$. MR-proADM above the cutoff value $(\geq 0.85)$ was associated with a fatal outcome (88.9 vs. $20.0 \%, p<0.01)$ and cardiovascular events (71.4 vs. $28.6 \%, p<0.01$ ); this association was not observed for hsTnT. Conclusion: Preoperative measurement of MR-proADM provides useful information for perioperative cardiac events in high-risk patients scheduled for noncardiac surgery.

(c) 2018 The Author(s)

Published by S. Karger AG, Basel

\section{Introduction}

In 2014, the European Society of Cardiology (ESC) and the European Society of Anesthesiology (ESA) produced guidelines for preoperative cardiac risk assessment and perioperative cardiac management of noncardiac surgery patients [1]. Several different strategies were used to identify patients who were at risk of major postoperative cardiovascular complications with preoperative risk assessment tools and, more recently, with the use of biomarkers [2, 3]. Midregional (MR) pro-adrenomedullin (proADM), the MR precursor of adrenomedullin (ADM), is of interest because it is an indicator of the severity of cardiovascular disease $[4,5]$. MR-proADM is a prime marker of global hemodynamic stress and cardiovascular dysfunction, and it is highly predictive of adverse outcomes in patients with heart failure [6]. Levels of MRproADM indicate significant cardiovascular risk factors in elderly patients [7] and can predict mortality in the general population [8]. In modern practice, the most currently used biomarkers are cardiac troponin C, troponin $\mathrm{I}$, and troponin $\mathrm{T}$ (TnT), because of their higher sensitivity and specificity compared with those of other biomarkers. Cardiac troponin I and TnT are proteins that are unique to the heart and are specific and sensitive biomarkers of myocardial damage [9]. The objective of this study was to evaluate the utility of preoperative MR-pro$\mathrm{ADM}$ and cardiac TnT for improved detection of patients at high risk for perioperative cardiac events and all-cause mortality after major noncardiac surgery.

\section{Subjects and Methods}

In this prospective, single-center, observational study, 90 patients undergoing major noncardiac surgery were recruited in 2013 at surgical clinics in the Clinical Center Nis, Serbia. The inclusion criteria were major noncardiac surgery (abdominal, thoracic, orthopedic, and vascular surgery), general anesthesia, age $>55$ years and at least one of the following cardiovascular risk factors: diabetes mellitus, hypertension, hyperlipidemia, active smoking, or a family history of cardiac disease. Exclusion criteria were emergency surgery; the inability to understand or sign an informed consent, and the lack of measurable levels of preoperative plasma MR-proADM (6 patients) and preoperative levels of highsensitivity TnT (hsTnT) (4 patients); 1 patient was lost as a result of surgery cancellation. In total, 79 patients were enrolled in the final analysis. Within the 14-day monitoring period, 14 patients developed a target event, and 65 were event free. The patients were also divided into groups of deceased patients ( 9 patients) and survivors (70 patients).

The Biomedical Ethics Committee of the Medical School (University of Nis) approved the study. Written informed consent was obtained from all participating patients. The study was carried out according to the principles of the Declaration of Helsinki.

Anesthetic management, perioperative care, and intensive care unit referral were provided at the discretion of the attending physicians. The attending clinicians were not blinded to the values of MR-proADM or hsTnT. The subjects were classified according to the physical status classification of the American Society of Anesthesiologists (ASA) [10]; the New York Heart Association (NYHA) classification [11] was recorded for each subject. During the perioperative period, adverse cardiac events, including the development or aggravation of congestive heart failure and acute myocardial infarction, as well as all-cause mortality within 14 days of surgery, were recorded. Peri- or postoperative myocardial infarction was diagnosed based on the universal definition of myocardial infarction [9]. To confirm the absence of myocardial damage, troponin was regularly assessed and electrocardiography or echocardiography was performed. The primary outcome was all-cause mortality, and secondary outcomes were defined as single or combined cardiovascular events during the perioperative period. The initial clinical assessment of patients included clinical history, physical examination, echocardiogram, blood tests, pulse oximetry, and chest X-ray. The follow-up period after surgery lasted until discharge or up to 14 days in the hospital. Blood samples were taken within $48 \mathrm{~h}$ prior to surgery from the antecubital vein and stored in serum Vacutainer tubes without additives. After centrifugation, the serum was separated and frozen at $-70^{\circ} \mathrm{C}$ until analysis. Cardiac hsTnT was measured in the serum specimens using a newly developed high-sensitive electrochemiluminescence immunoassay (hsTnT) on a Cobas e411 analyzer (Roche Diagnostics $\mathrm{GmbH}$, Mannheim, Germany) according to the recommendations of the manufacturer (expressed as ng/L). MR-proADM (expressed as nmol/L) was detected in serum with a "sandwich" immunoluminometric assay (MR-proADM; Brahms AG, Hennigsdorf, Germany).

\section{Statistical Analyses}

Statistical analyses were done with the Statistical Package for the Social Sciences (SPSS 16.0 for Windows; SPSS Inc., Chicago, IL, USA). All data are presented as means and standard deviations, medians, and interquartile ranges, or percentages. Comparisons between surviving and deceased patients, and patients with and without any cardiovascular event were made using the Student $t$, Mann-Whitney, or $\chi^{2}$ tests. Receiver-operating characteristic (ROC) curve analysis was done to determine the optimal threshold 
Table 1. Demographic and clinical characteristics in relation to death rate and occurrence of cardiovascular events

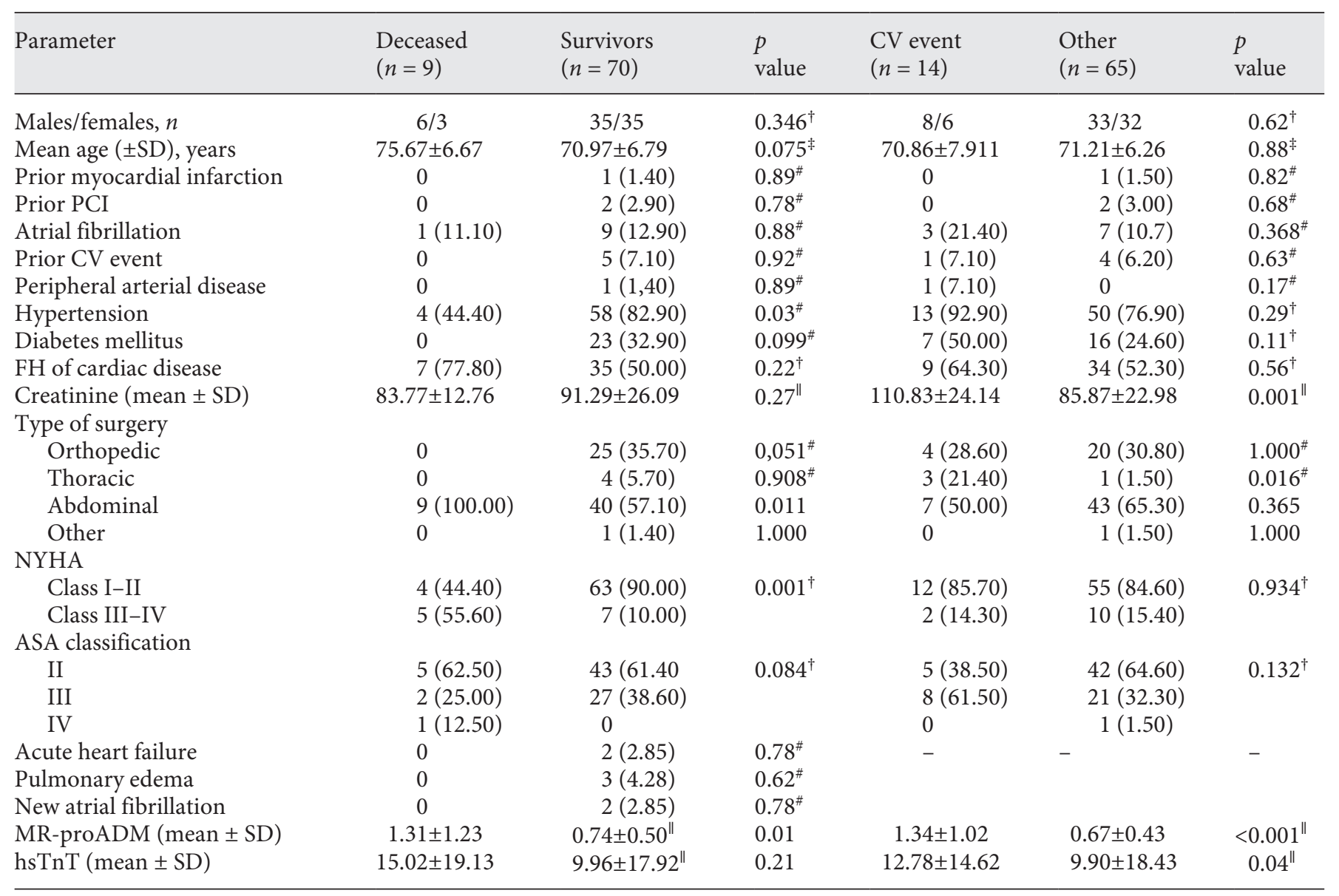

Numbers (\%) are shown unless indicated otherwise. PCI, percutaneous coronary intervention; CV, cardiovascular; FH, family history; MR-proADM, midregional pro-adrenomedullin; hsTnT, high-sensitivity troponin T; NYHA, New York Heart Association; ASA, American Society of Anesthesiologists. ${ }^{\dagger} \chi^{2}$ test; ${ }^{*} \mathrm{t}$ test; ${ }^{\|}$Mann-Whitney test; ${ }^{\#}$ Fisher exact test.

with a maximum combination of specificity and sensitivity in predicting mortality. Survival curves were estimated by the KaplanMeier method; the differences were assessed with the log-rank test. Differences in the proportions of patients in the upper quartile ranges for MR-proADM and hsTnT between defined groups were analyzed by $\chi^{2}$ test. A value of $p<0.05$ was considered statistically significant.

\section{Results}

During monitoring, 14 (17.72\%) patients developed a target event, and $65(82.28 \%)$ were event free. In 9 patients, the outcome was death $(11.39 \%) ; 70$ patients (88.61\%) survived.

Preoperative MR-proADM and

Perioperative Cardiovascular Mortality

\section{Mortality}

The mean age of the patients was $71.29 \pm 6.62$ years (range: 55-87), with no difference between the deceased and survivor groups. The incidence of hypertension was higher in patients who survived $(82.90 \%)$ than in those who died $(44.40 \%, p=0.03)$. The incidence of abdominal surgery was significantly more frequent in patients who died ( 100.0 vs. $57.1 \%, p=0.01)$; thus, all deceased patients underwent this type of surgery. The incidence of antiplatelet treatment was statistically higher in deceased patients than in those who survived ( 66.7 vs. $14.3 \%, p<0.01$ ) (data not shown). NYHA class III-IV was significantly more frequent in deceased than in surviving patients (55.6 vs. $10.0 \%, p<0.001$ ). The other demographic and 


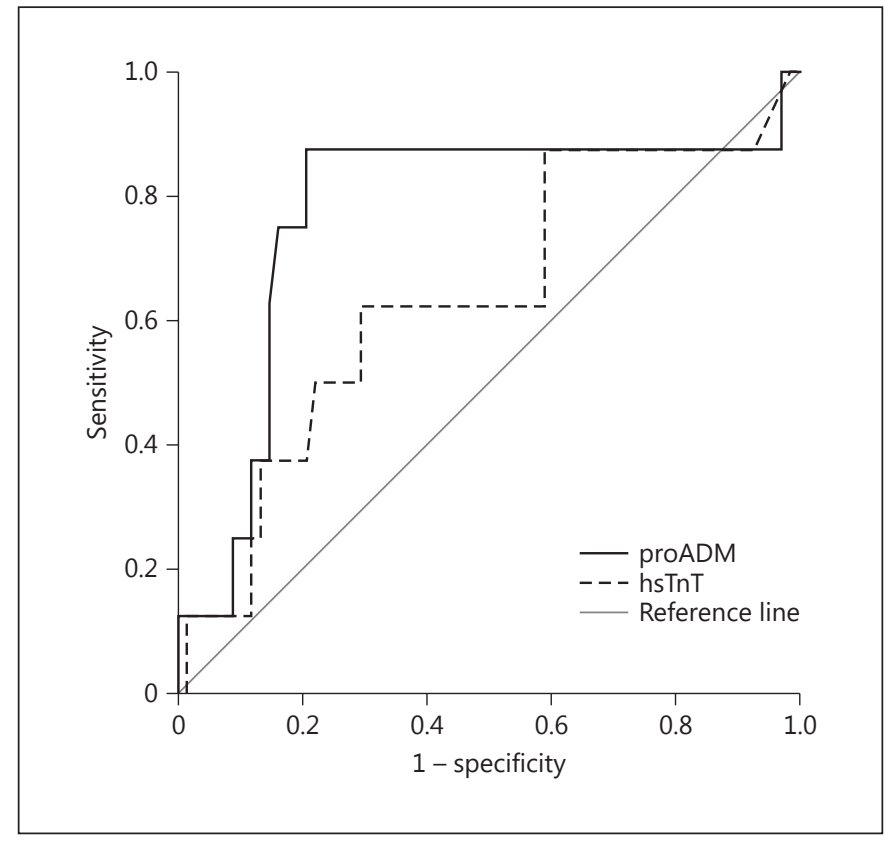

Fig. 1. ROC curve for midregional pro-adrenomedullin (MRproADM) and high-sensitivity troponin $\mathrm{T}$ (hsTnT).

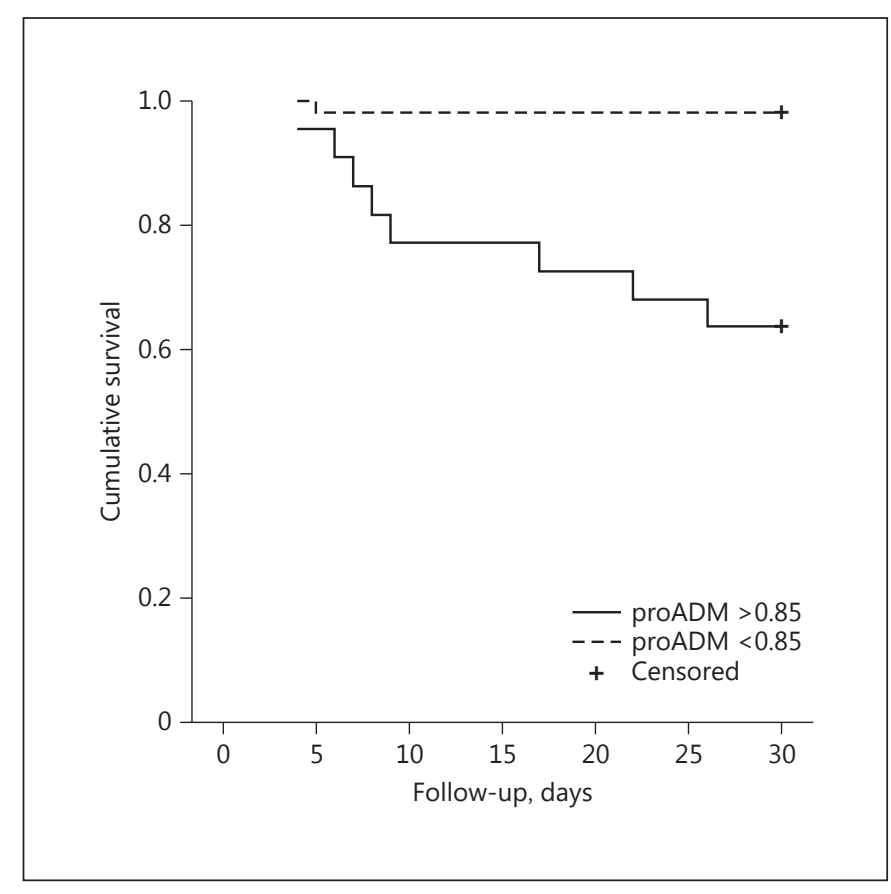

Fig. 2. Kaplan-Meier survival rate curve in relation to the values of midregional pro-adrenomedullin (MR-proADM; cutoff: 0.85 ).

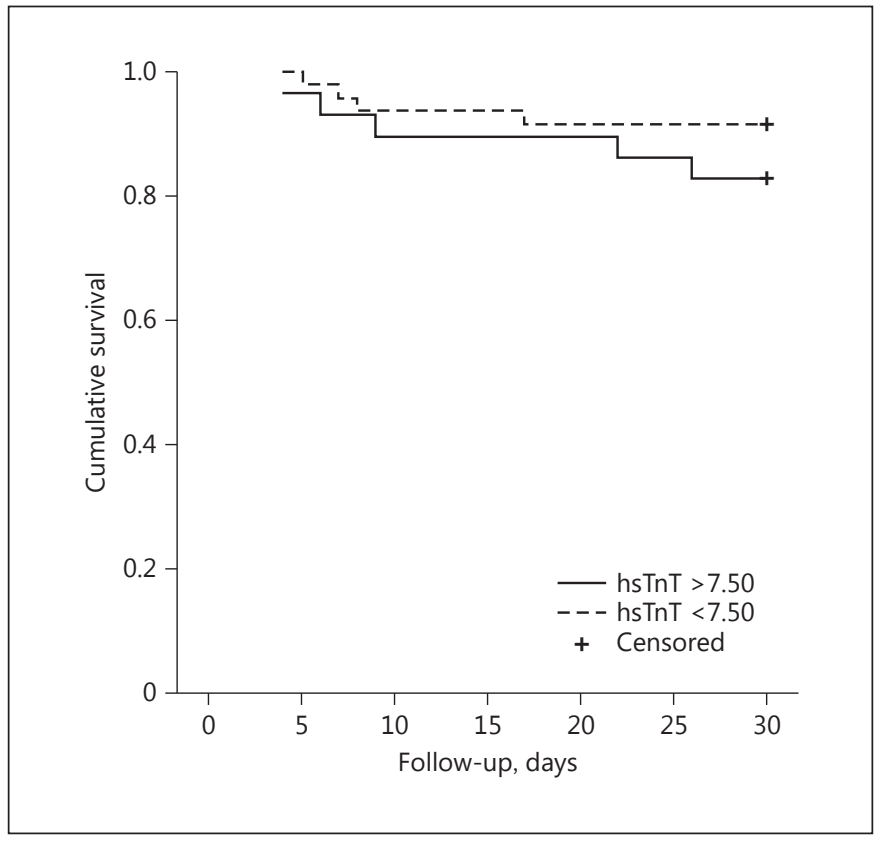

Fig. 3. Kaplan-Meier survival rate curve in relation to the values of high-sensitivity troponin T (hsTnT; cutoff: 7.50).

clinical parameters did not significantly differ between the study groups (Table 1).

The concentration of MR-proADM was significantly higher in deceased than in surviving patients $(1.31 \pm 1.23$ vs. $0.74 \pm 0.50 \mathrm{nmol} / \mathrm{L}, p=0.01)$. hsTnT levels did not significantly differ between the study groups $(15.02 \pm$ 19.13 vs. $9.96 \pm 17.92 \mathrm{ng} / \mathrm{L}, p=0.21$ ).

The discriminatory potential for death outcome was higher for MR-proADM (AUC $=0.77, p=0.01$ ) than hsTnT. The calculated cutoff value was 0.85 , with a sensitivity of $87.50 \%$ (Fig. 1 ) and a specificity of $79.40 \%$. For hsTnT, the calculated cutoff value was 7.50 , with a sensitivity of $62.50 \%$ and a specificity of 64.70 . The AUC for hsTnT was $0.64(p=0.21)$.

From the cutoff value determined in the ROC analysis, groups were formed in relation to the concentrations of MR-proADM $(\geq 0.85)$ and hsTnT $(\geq 7.50)$. Patients with MR-proADM values $>0.85$ had a significantly shorter survival rate than did patients with lower values (median survival [SE]: 29.54 [0.45] vs. 23.59 [2.06], $p<0.001$; Fig. 2). hsTnT levels did not affect the survival rate $(p=$ 0.25 ). The upper quartile of age and hsTnT was not associated with a fatal outcome (Table 2). The upper quartile of MR-proADM was significantly associated with a fatal outcome ( 88.9 vs. $20.0 \%, p<0.01)$ and cardiovascular events $(71.4$ vs. $28.6 \%, p<0.01)$. hsTnT level above the 
Table 2. Interquartile analysis according to age, midregional pro-adrenomedullin (MR-proADM), and high-sensitivity troponin $\mathrm{T}$ (hsTnT) values

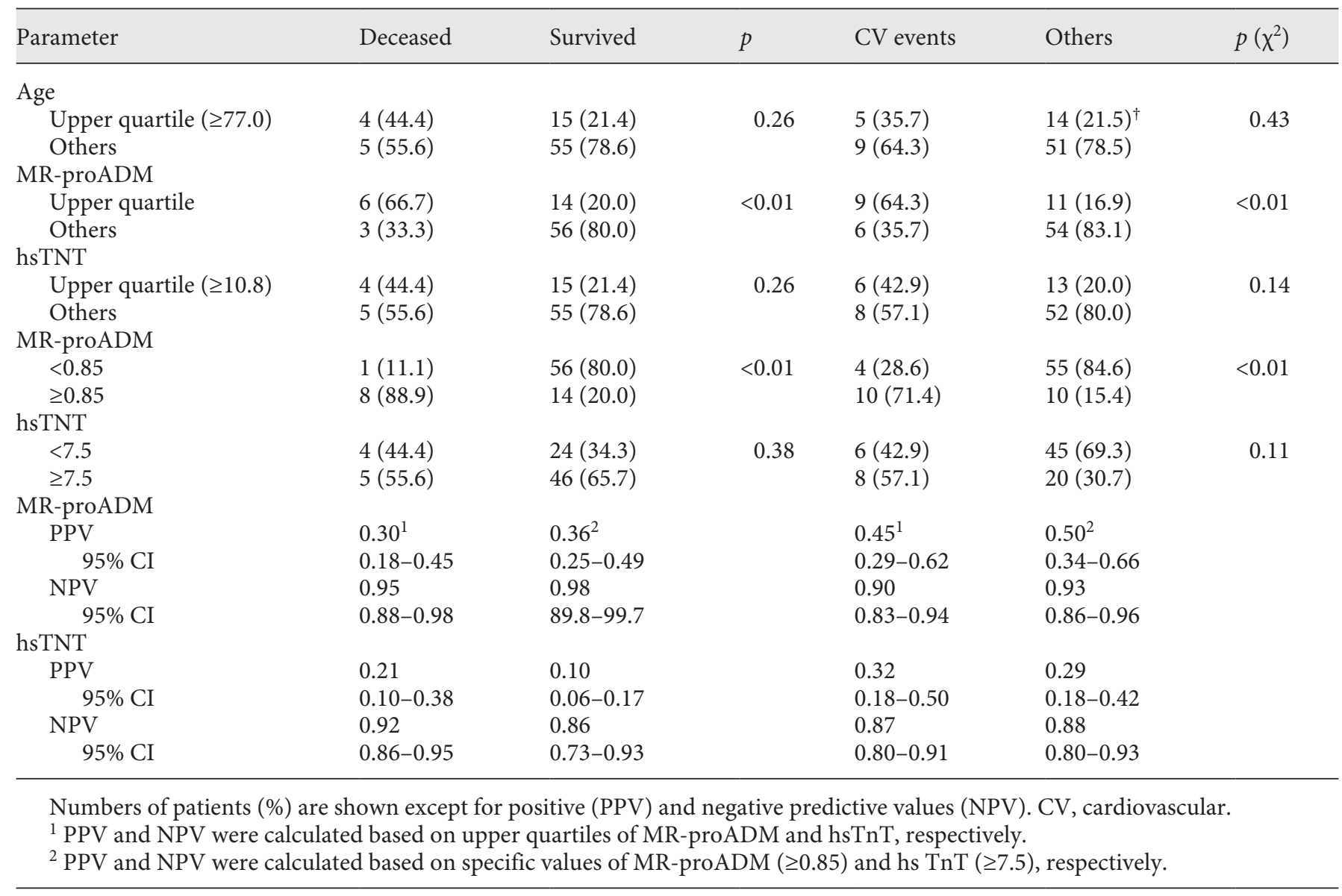

cutoff was neither significantly associated with a fatal outcome $(p=0.38)$ nor with cardiovascular events $(p=$ 0.11 ; Fig. 3).

\section{Target Events}

Creatinine levels were higher in patients with any cardiovascular event than in the event-free group (110.83 \pm 24.14 vs. $85.87 \pm 22.98 \mu \mathrm{mol} / \mathrm{L}, p=0.001)$. Thoracic surgery was performed more frequently in patients with cardiovascular events ( 21.4 vs. $1.5 \%, p=0.01)$. The values of the other biochemical and clinical parameters did not significantly differ in relation to the occurrence of any cardiovascular event (Table 1). All of the patients who died were in the intensive care unit. The patients who died were significantly more often admitted to intensive care units (Fisher test: $p=0.034$ ). The deceased patients spent on average more days in the intensive care unit than the surviving patients (5.29 \pm 3.65 days vs. $2.21 \pm 1.75, p=0.003$ ).

\section{Discussion}

Our study showed that MR-proADM was a better parameter than hsTnT in identifying subgroups of patients who are at an extremely high risk for peri- or postoperative adverse cardiac events and all-cause mortality. We suggest that the use of MR-proADM in risk stratification could identify high-risk patients who might benefit from risk reduction strategies.

The total high perioperative mortality of $11.39 \%$ and cardiovascular morbidity of $6.33 \%$ in this study can partly be explained by the facts that the patients included in our study were elderly ( $71.29 \pm 6.62$ years), that the study group was relatively small, that there was a great number of comorbidities, and that extensive noncardiac surgeries carry a particularly high risk. All deceased patients were over 65 years old $(75.67 \pm 6.67)$. All of the patients who died were subjected to abdominal (100\%), mostly radical, 
resections. The facts that the incidence and mortality rates of malignancy have increased since 1999 and that the mortality rate in the population older than 65 years is significantly higher than that observed in other countries of Europe may further explain the high mortality rate found here [12]. In older patients who live in rural parts of the country, have low monthly earnings, and/or are detached from family members, access to the health care network requires more efforts. These were the main reasons for establishing a 14-day follow-up and a routine postoperative physical examination that was conducted after 2 weeks by surgeons [13]. Unlike developed countries, our patients were diagnosed with a higher stage of disease during the nonexistence of organized national screening at the time of this research, with an insolvent health care system and low health care consumption per capita $[14,15]$.

Over the last decade, numerous studies have increased our knowledge on the role of biomarkers as predictors of mortality and cardiovascular morbidity in noncardiac surgery [16-19]. MR-proADM was identified as a novel predictor of mortality in surgical patients [20]. Risk prediction was further improved by combined biomarker analysis [2]. Several studies have examined the prognostic utility of MR-proADM in nonsurgical patients [21-23]. Recent studies have confirmed the use of hsTnT for the risk stratification of patients undergoing noncardiac surgeries $[24,25]$. We did not observe an association of preoperative values of hsTnT and short-term mortality, as reported by Devereaux et al. [26] who concluded that an association exists between peak levels of postoperative hsTnT and 30-day mortality. We suggest that it was an impact of surgical stress response mechanisms. MR-pro$\mathrm{ADM}$ has better discriminatory potential than hsTnT, and because it is strongly associated, it could be used as a predictor of perioperative death and cardiovascular events in our study.

Assuming that all deceased patients underwent abdominal surgery and that patients who underwent thoracic surgery developed cardiovascular events more frequently, with all of them having high MR-proADM, it could be considered a marker independent of the underlying pathological process. This is most likely related to its response to different systemic cellular and metabolic damage. Earlier studies focused on a role of MR-proADM and hs TnT in heart diseases. There is an association between TnT levels in malignant patients without cardiac diseases and adverse mortality [27, 28]. MR-proADM, which is secreted by malignant cells [29], and hsTnT were shown to be independent predictors of mortality in a het- erogenic group of oncological patients prior to induction of anticancer therapy [30]. The same study demonstrated a relationship among MR-proADM, the proinflammatory cytokine IL-6, and the inflammatory marker C-reactive protein. This presents a possible explanation for its good prognostic value in patients with different surgical treatments and pathological conditions.

\section{Conclusion}

Patients at high risk for perioperative adverse cardiovascular events and all-cause mortality can be identified by a single preoperative measurement of MR-proADM. We conclude that preoperative MR-proADM was strongly associated with mortality and adverse outcomes in this group of surgical patients. The advantages of this method include that it is noninvasive, objective, and rapid. In addition, it provides more reliable and prompt information to physicians regarding clinical risk factors than the measurement of hsTnT. Since this was a single-center study, further research in a larger cohort of patients is needed to make clinically applicable conclusions.

\section{Acknowledgments}

The authors wish to thank the attending physicians Vladan Cvetanovic, Nenad Jovanovic, and Sonja Stamenic, without their efforts this study would not have been possible.

\section{Disclosure Statement}

The authors have no conflict of interest to disclose.

References 
-3 Leibowitz D, Planer D, Rott D, et al: Brain natriuretic peptide levels predict perioperative events in cardiac patients undergoing noncardiac surgery: a prospective study. Cardiology 2008;110:266-270.

4 Peacock WF: Novel biomarkers in acute heart failure: MR-pro-adrenomedullin. Clin Chem Lab Med 2014;52:1433-1435.

5 Schnabel RB, Schulz A, Messow CM, et al: Multiple marker approach to risk stratification in patients with stable coronary artery disease. Eur Heart J 2010;31:3024-3031.

-6 Pousset F, Masson F, Chavirovskaia O, et al: Plasma adrenomedullin, a new independent predictor of prognosis in patients with chronic heart failure. Eur Heart J 2000;21:10091014.

7 Eggers KM, Venge P, Lindahl B, et al: Associations of mid-regional pro-adrenomedullin levels to cardiovascular and metabolic abnormalities, and mortality in an elderly population from the community. Int J Cardiol 2013; 168:3537-3542.

-8 Funke-Kaiser A, Havulinna AS, Zeller T, et al: Predictive value of midregional pro-adrenomedullin compared to natriuretic peptides for incident cardiovascular disease and heart failure in the population-based FINRISK 1997 cohort. Ann Med 2014;46:155-162.

$>9$ Thygesen K, Mair J, Katus H, et al: Recommendations for the use of cardiac troponin measurement in acute cardiac care. Eur Heart J 2010;31:2197-2204

10 Owens WD, Felts JA, Spitznagel EL Jr: ASA physical status classifications: a study of consistency of ratings. Anesthesiology 1978;49: 239-243.

11 The Criteria Committee of the New York Heart Association: Nomenclature and Criteria for Diagnosis of Diseases of the Heart and Great Vessels, ed 9. Little, Brown, Boston, 1994, pp 253-256.

12 Mihajlovic J, Pechlivanoglou P, MiladinovMikov, et al: Cancer incidence and mortality in Serbia 1999-2009. BMC Cancer 2013;13: 18.
13 Urosevic J, Odovic G, Rapaic D, et al: Quality of life of the elderly in urban and rural areas in Serbia. Vojnosanit Pregl 2015;72:968-974.

14 Altobelli E, D’Aloisio F, Angeletti PM: Colorectal cancer screening in countries of European Council outside of the EU-28. World J Gastroenterol 2016;22:4946-4957.

15 Scepanovic M, Jovanovic O, Keber D, et al: Faecal occult blood screening for colorectal cancer in Serbia: a pilot study. Eur J Cancer Prev 2017;26:195-200.

16 Filipovic M, Jeger R, Probst C, et al: Heart rate variability and cardiac troponin I are incremental and independent predictors of oneyear all-cause mortality after major noncardiac surgery in patients at risk of coronary artery disease. J Am Coll Cardiol 2003;42: 1767-1776.

17 van Waes JA, Nathoe HM, de Graaff JC, et al: Myocardial injury after noncardiac surgery and its association with short-term mortality. Circulation 2013;127:2264-2271.

18 Devereaux PJ, Chan MT, Alonso-Coello P, et al: Association between postoperative troponin levels and 30-day mortality among patients undergoing noncardiac surgery. JAMA 2012;307:2295-2304.

19 Rodseth RN, Padayachee L, Biccard BM: A meta-analysis of the utility of pre-operative brain natriuretic peptide in predicting early and intermediate-term mortality and major adverse cardiac events in vascular surgical patients. Anaesthesia 2008;63:1226-1233.

20 Csordas A, Nietlispach F, Schuetz P, et al: Midregional proadrenomedullin improves risk stratification beyond surgical risk scores in patients undergoing transcatheter aortic valve replacement. PLoS One 2015;10: e0143761.

21 Haaf P, Twerenbold R, Reichlin T, et al: Midregional pro-adrenomedullin in the early evaluation of acute chest pain patients. Int J Cardiol 2013;168:1048-1055.
22 Khan SQ, O’Brien RJ, Struck J, et al: Prognostic value of midregional pro-adrenomedullin in patients with acute myocardial infarction: the LAMP (Leicester Acute Myocardial Infarction Peptide) study. J Am Coll Cardiol 2007;49:1525-1532.

23 Wild PS, Schnabel RB, Lubos E, et al: Midregional proadrenomedullin for prediction of cardiovascular events in coronary artery disease: results from the AtheroGene Study. Clin Chem 2012;58:226-236.

24 Biccard B, Naidoo P, de Vasconcellos K: What is the best pre-operative risk stratification tool for major adverse cardiac events following elective vascular surgery? A prospective observational cohort study evaluating pre-operative myocardial ischaemia monitoring and biomarker analysis. Anaesthesia 2012;67: 389-395.

25 Weber M, Luchner A, Seeberger M, et al: Incremental value of high-sensitive troponin $\mathrm{T}$ in addition to the revised cardiac index for peri-operative risk stratification in non-cardiac surgery. Eur Heart J 2013;34:853-862.

26 Devereaux PJ, Biccard BM, Sigamani A, et al: Association of postoperative high-sensitivity troponin levels with myocardial injury and 30-day mortality among patients undergoing noncardiac surgery. JAMA 2017;317:16421651.

27 Lim E, Li Choy L, Flaks L, et al: Detected troponin elevation is associated with high early mortality after lung resection for cancer. J Cardiothorac Surg 2006;1:37.

28 Danese E, Montagnana M, Giudici S, et al: Highly-sensitive troponin I is increased in patients with gynecological cancers. Clin Biochem 2013;46:1135-1138.

29 Nikitenko LL, Fox SB, Kehoe S, et al: Adrenomedullin and tumour angiogenesis. Br J Cancer 2006;94:1-7.

30 Pavo N, Raderer M, Hülsmann M. et al: Cardiovascular biomarkers in patients with cancer and their association with all-cause mortality. Heart 2015;101:1874-1880.
Preoperative MR-proADM and

Perioperative Cardiovascular Mortality
Med Princ Pract

DOI: $10.1159 / 000488197$ 